\title{
Biomedical Imaging: Contrast Agents and Multifunctionality
}

\section{Mohammad E Khosroshahi*}

MIS-Electronics, Nanobiophotonics and Biomedical Research Lab., Richmond Hill, Canada

\section{Bioimaging}

Bioimaging is one of the major streamlines of comprehensive cancer care in both diagnosis and research with significant advantages of real time monitoring, minimal or no invasive action, and operational over relatively wide ranges of time and size scales involved in biological and pathological processes. Bioimaging plays a great role in different stages of cancer management: Prediction, screening, biopsy for detection, staging, prognosis, therapy planning, therapy guidance and therapy response [1-5]. Biomarkers identified from the genome and proteome can be selectively targeted and chemical binding can improve their imaging signal. Various pharmaceutical therapies that have been developed for cancer are classified as cytotoxic, antihormonal, immunotherapeutic and molecular targeted. Imaging can help the molecular targeted therapies to control their effectiveness and include: signal transduction, angiogenesis, cell cycle inhibitors, apoptosis inducers and epigenetic modulators [6].

The molecular imaging is a combined functional and structural imaging modality, which effectively can be used to achieve the health benefit from understanding the spatial mapping at the whole-body level and molecular processes within cells and tissues [7]. Various targeted agents for cancer markers are for example: epidermal growth factor receptor (EGFR), $\alpha_{v} \beta_{3}$ integrin, vascular endothelial growth factor (VEGF), carcinoembryonic antigen (CEA) and folate receptors (FR). Clearly, the development of minimally invasive targeted therapy and drug delivery should be based on the guided imaging system. Most clinical imaging systems are based on the interaction of electromagnetic radiation with body tissues and fluids except ultrasound which is based on the reflection, scattering and the frequency shifts of acoustic waves (i.e., Doppler effect). Ultrasound also has the capability of imaging tissue elasticity, thus can be employed in differential diagnosis of breast cancer, prostate cancer, and liver fibrosis because cancer tissues are less elastic than normal tissue and ultrasound elastography $[8,9]$. High frequency electromagnetic radiation such as $\gamma$-rays, X-rays or UV light is ionizing, which can cause mutation hence leading to cancer. In contrast, non-ionizing radiation imaging systems including IR spectroscopy, microwave imaging spectroscopy, photoacoustic and thermoacoustic imaging which are readily used for imaging poses no such danger. Imaging systems has one common point; they vary in physical properties including sensitivity, temporal and spatial resolution. In addition, the imaging systems produce images that have differences in contrast. The differences in contrast can be due to changes in physical properties caused by the endogenous nature of the tissue e.g.: radiation absorption, reflection, transmission, magnetic relaxivity, magnetic susceptibility, oxygenation, spectral distribution, electrical impedance, mechanical elasticity, etc. or exogenous mechanisms e.g.: radiation absorption, reflection, emission, magnetic relaxivity, magnetic susceptibility, isotope spectra, fluorescence, perfusion, hypoxia, etc. Finally, it is believed that the sensitivity and specificity of diagnostic systems can be improved by combining the systems as one system known as multimodal system.

\section{Multimodal Strategies}

No single imaging system can provide all the required information in biomedical imaging technology. As mentioned above each imaging modality has its own advantages and limitations in terms of sensitivity, resolution, accuracy and quantitative capabilities. The major problem which is often faced with single-modality imaging is the inability to assure the conformance of diagnosis, which is very important factor in determining the treatment. The problem is solved by multimodal imaging system where a combination of techniques with complementary strengths offer unique benefits which are not met by individual methods. Therefore, utilizing such complementary imaging modes will greatly improve the diagnosis and treatment reliance and render extra comfort to both physicians and patients. Multimodal imaging techniques can be obtained in two different approaches (i) combining the different imaging instruments into one unit, (ii) to develop multimodal imaging agents.

\section{Examples of combined imaging systems}

$$
\begin{array}{ll}
\text { - } & \text { PET-CT } \\
\text { - } & \text { MRI-Optical } \\
\text { - } & \text { MRI-PET } \\
\text { - } & \text { MRI-PET-NIRF } \\
\text { - } & \text { MRI/SPECT } \\
\text { - } & \text { MRI-PA-Raman } \\
\text { - } & \text { SPECT-CT } \\
\text { - } & \text { PA-US } \\
\text { - } & \text { PA-OCT } \\
\text { - } & \text { PA-Fluorescence } \\
\text { - } & \text { PA-US-Agents. }
\end{array}
$$

\section{Examples of multimodal imaging agents}

Although every individual imaging modality system has its particular contrast agents, multimodal imaging systems also require its customized multimodal contrast agents. Some examples are as follows:

Iron oxide-Gold nanoparticles (SPION-Au): has been used in MRI-PAI dual mode imaging [10] where they showed the defined structural characteristics and physical properties of this agent not only

*Corresponding author: Mohammad E Khosroshahi, MIS-Electronics, Nanobiophotonics and Biomedical Research Lab., Richmond Hill, Canada, Tel: 14169781287; E-mail: Khosrom@mie.utoronto.ca

Received: February 09, 2017; Accepted: February 21, 2018; Published: February 23, 2018

Citation: Khosroshahi ME (2018) Biomedical Imaging: Contrast Agents and Multifunctionality. J Nanomed Nanotechnol 9: 485. doi: 10.4172/21577439.1000485

Copyright: @ 2018 Khosroshahi ME. This is an open-access article distributed under the terms of the Creative Commons Attribution License, which permits unrestricted use, distribution, and reproduction in any medium, provided the original author and source are credited. 
offer contrast for electron microscopy and MRI but also a new mode of magneto photoacoustic imaging. The (Au-SPION) provides a superior contrast compared to using only single conventional nanoparticles. It was recently shown that the combined imaging system based on interferometric scattering (iSCAT) and AFM enhances the accuracy, temporal resolution and capable of producing high resolution images of individual SPION-Au nanoparticles [11].

Iron oxide-gold nanoclusters: Small nanoclusters with optical, magnetic, and therapeutic functionality, designed by assembly of nanoparticle building blocks, offer broad opportunities for targeted cellular imaging, therapy, and combined imaging and therapy [12]. Approximately $30 \mathrm{~nm}$ stable uniformly sized near-infrared (NIR) active, superparamagnetic nanoclusters formed by kinetically controlled self-assembly of gold-coated iron oxide nanoparticles. The nanoclusters of approximately $70 \mathrm{~nm}$ iron oxide primary particles with thin gold coatings display intense NIR (700-850 nm) absorbance.

Single-walled carbon nanotubes (SWNTs): New size-controlled and biocompatible Gd2)3-DEG-gelatin nanoparticles were fabricated for MRI and PA [13]. Single-walled carbon nanotubes (SWNTs) is another multimodal contrast agent which possess surface properties and biocompatibility similar to plasmonic nanoparticles with wide absorption that can produce strong photoacoustic signals.

Perfluorocarbon (PFC) droplets: Phase-change contrast agents transform liquid emulsions into microbubbles (MBs) contrast agents that can have both diagnostic and therapeutic functions. PFC droplets undergo a volumetric expansion when is subject to sufficient acoustic pressures delivered by an ultrasound transducer, called acoustic droplet vaporization (ADV) [14]. Similarly, when it is subject to optical irradiation, vaporization also occurs (ODV) [15]. Thus, when the laser fluence is below the vaporization threshold, the droplets remain in the liquid phase and can be used as a PA contrast agent. However, when the fluence exceeds the threshold, it induces droplet vaporization resulting in $\mathrm{MBs}$ which can be used for contrast enhanced ultrasound imaging. MBs can be composed of phospholipids, albumin or polymer. These gas-filled can produce strong acoustic scattering relative to the surrounding tissue. In more advance form, Au MBs comprise of albumin-shelled microbubbles with encapsulated gold nanorods and have been used as PA/US dual modality contrast agent for thermotherapy [16].

Dye-doped PFC nanoparticles: These contrast agents have been used as PAI-FI [17]. Spectroscopic characterization of the developed NIR dye-loaded perfluorocarbon-based nanoparticles for combined fluorescence and PA imaging revealed distinct dye-dependent photo physical behavior. They demonstrated that the enhanced contrast allows detection of regional lymph nodes of rats in-vivo with timedomain optical and photoacoustic imaging methods. Also, the use of fluorescence lifetime imaging provided a strategic approach to bridge the disparate contrast reporting mechanisms of fluorescence and PA imaging methods.

\section{Multi-functionality}

The progress and advances in nanobiotechnology during the last decade has resulted in significant achievements. On the hand, it seems, the everyday increasing clinical demands has a direct role in shaping, directing and even accelerating part of this progress in specific directions. For example, the heterogenicity of cancers necessitates image-guided therapies, where personalized disease treatments are planned based on individual patients' pathological conditions and responses to the treatment. Consequently, as a result of such demands, the idea of multifunctional (i.e., multimoiety) nanoparticles was formed. Multifunctional nanoparticles are those that are capable of combining various functional features in one package acting as a single multifunctional nanoprobe for different purposes: detection, imaging, therapy or drug delivery $[18,19]$. Though, the clinical demands provided the necessary motivation it is, however, important to acknowledge the fact that emergence of new advance nanoparticles itself is due to nanofabrication techniques advancement which render various possibilities for biomedical applications. Basically, a nanoparticle outer layer (normally with a suitable coating) can be linked to a specific targeting ligand that only recognizes the unique features of the surface of the target. In addition, other moieties can be attached simultaneously for other purposes including imaging and drug delivery. Therefore, the function of such multifunctional structure depends on the application which in turn governs the type of components that can be used.

\section{Conclusion}

Let us remind that the high surface area-to-volume ratio facilitates surface loading capability with more cargoes such as targeting moieties, imaging and therapeutic agents or other functional molecules. Once such a multifunctional structure is administrated to body, it can be triggered by light excitation (optically and thermally) or by magnetic field for diagnostic and therapeutic applications. However, prior to their use, a number of requirements ought to be satisfied: (a) biocompatibility, (b) non-toxicity, (c) stability in in-vivo environment, (d) high selectivity, (e) long circulation time in the bloodstream and (f) efficient clearance by the renal system. The next stage to consider is the synthesis process. The synthesis of nanoparticles has received a great deal of interest and attention because of their potential biomedical applications which in turn are affected by the kind of surface modification. There are broadly two types of modification methods: chemical and physical where the former offers a stronger and robust bonding and stable surface ligand. A proper surface modification determines how well biomolecules are conjugated on the nanoparticles. A wide variety of contrast agents and labels is required for different types of detection and imaging such as MRI, PET, SPECT and fluorescence-based imaging. While the last method is widely used for in-vitro imaging, the others are generally applied for in-vivo applications. An important class of nanoparticles comprises those made of inorganic materials such as metals, metal oxide, semiconductor, rare earth minerals and silica. These materials possess unique electrical, optical, magnetic and plasmonic properties due to quantum mechanical effects at nanoscale. Most nanocrystals can be fabricated with a great control on size, shape, composition and physical properties. Potential single agents and functional nanoparticles that can be used for both therapy and bioimaging are: Dyes (for example, Indocyanine-green(ICG), Fluorescein isothiocyanate (FITC), Alexa Fluor 750, Evans blue, IR Dye 800CW, Methylene blue,...), gold nanoparticles, gold nanoshells, gold nanorods, nanocages, nanostars, nanobeacons, SPIONS, polymeric NPs (e.g., PLA, PEG, PEO, PPO and PLGA), dendrimers, Silica, QDs and upconversion nanoparticles. Multimodal nanoparticles can often offer better spatial registration of different imaging modality and avoid excessive immune response caused by repeated challenge. A number of multimodal nanoparticles combine the use of a full body scan with an imaging modality that offers local images with higher resolution [20]. This technique allows characterization of disease at multiple spatial scales. For example, SPIONs labeled with Cy5.5, functionalized silica nanoparticles or FITC-conjugated magnetite nanoparticles and many other similar combinations were utilized for biomedical imaging applications [21-23]. 
Citation: Khosroshahi ME (2018) Biomedical Imaging: Contrast Agents and Multifunctionality. J Nanomed Nanotechnol 9: 485. doi: 10.4172/21577439.1000485

\section{References}

1. Kent MS, Port JL, Altorki NK (2004) Current state of imaging for lung cancer staging. Thoractic Surg Clinc 14: 1-13.

2. Lee K, Jeong Y, Han J (2004) T1 non-small cell lung cancer: imaging and histopathologic findings and their prognostic implications. Radiograph 24: 1617-1636.

3. Feme C (2005) Role of imaging to choose treatment. Cancer imaging 5 : S113-S119.

4. Lehman CD, Isaacs C, Schnall MD, Pisano ED, Ascher SM, et al. (2007) Cancer yields of mammography, MR, and US in high risk women: prospective multi-institution breast cancer screening study. Radiology 244: 381-388.

5. Brindle K (2008) New approaches to imaging tumour responses to treatment. Nature Rev. Cancer 8: 94-107.

6. Brindle K (2003) Molecular imaging using magnetic resonance: new tools for the development of tumour therapy. British J Radiolog 76: S111-S117.

7. Weber J, Beard P, Bohndiek S (20116) Contrast agents for molecular photoacoustic imaging. Nature Methods 13: 639-650.

8. Lerner R, Huang S, Parker S (1990) Sonoelasticity images derived from ultrasound signals in mechanically vibrated tissues. Ultrasound in Med and Biolog 16: 231-239.

9. Pallwein L, Mitterberger M, Struve $P$ (2007). Real-time elastography for detecting prostate cancer: preliminary experience. British J Ult Int 100: 42-46.

10. Jin Y, Jia C, Huang S, Gao X (2010) Multifunctional nanoparticles as coupled contrast agents. Nat Commun 1: 1-8.

11. Khosroshahi ME, Ghazanfari L, Hasannejad Z (2017) Dual mode atomic force microscopy and interferometric scattering imaging of single $\mathrm{Fe}_{3} \mathrm{O}_{4} @ \mathrm{Au}$ nanoshell synthesized for biomedical applications. Nano Res Appl 3: 1-5.

12. Ma L, Feldman M, Tam J, Paranjape A (2009) Small multifunctional nanoclusters for targeted cellular imaging and therapy. ACs Nano. 3: 2686-2696.
13. Kimura $Y$, Kamisugi R, Narazaki M (2012) Size-controlled and biocompatible $\mathrm{Gd}_{2} \mathrm{O}_{3}$ nanoparticles for dual photoacoustic and MRI imaging. Adv Healthc Mater 1: 657-660.

14. Kripfgans O, Fowlkes J, Miller D (2000) Acoustic droplet vaporization for therapeutic and diagnostic applications. Ultr Med Biolo 26: 1177-1189.

15. Strohm E, Rui M, Kolios M, Gorelikov I (2010) Optical droplet vaporization photoacoustic characterization of perflurocarbon droplets. IEEE Int Ult Symp 449-498.

16. Wang Y, Liao A, Chen J, Wang C, Li P (2012) Photoacoustic/ultrasound dualmodality contrast agent and its application to thermotherapy. J Biomed Opt 17: 045001

17. Akers W, Kim C, Berezin M (2010) Non-invasive photoacoustic fluorescent sentinel lymph node identification using dye-loaded perfluorocarbon nanoparticles. ACS nano 5: 173-182.

18. Khosroshahi ME, Tajabadi M (2017) Multifunctional nanoplatform for targeted laser-induced hyperthermia and microscopy of breast cancer cells using SPION-based gold and folic acid conjugated nanodendrimers: An in vitro Assay. J Nanomed Nanotech 8: 2.

19. Mallick A, More P, Ghosh S (2015) Dual drug conjugatd nanoparticle for simultaneous targeting of mitochondria and nucleous in cancer. ACS 7: 75847598

20. Bao G, Mitragotri S, Tong SH (2013) Multifunctional nanoparticles for drug delivery and molecular imaging. Annu Rev Biomed Eng. 15: 253-282.

21. Jin J, Hu-Jang L, Sangyong J, Sang Y (2013) Distribution and accumulation of Cy5.5-labeled thermally cross-linked superparamagnetic iron oxide nanoparticles in the tissues of ICR mice. J Vet Sci 14: 473-479.

22. Gomes M, Cunha A, Trindade T (2016) The role of surface functionalization of silica nanoparticles for bioimaging. J Innovative Opt Health Sci 9: 1630005-1-16.

23. Khosroshahi ME, Asemani M (2017) Synthesis, characterization and imaging of fluorescein isothiocyanate conjugated magnetite nanoparticles in MCF 7 breast cancer cell line. Int J Nanomat Nanotech Nanomed 3: 044-050. 